\title{
Mitral valve replacement in a patient with infective endocarditis and aneurysm of the cerebral artery: A case report
}

\author{
Senka Mesihović-Dinarević ${ }^{1}$, Mirza Halimić ${ }^{1}$, Zijo Begić1 ${ }^{1}$, Almira Kadić ${ }^{1}$, \\ Mirsad Kacila ${ }^{2}$, Edin Omerbašić ${ }^{2}$, Nusreta Hadžimuratović ${ }^{2}$, Eldin Burazerović ${ }^{3}$
}

${ }^{1}$ Paediatric clinic, University Clinical Centre Sarajevo

${ }^{2}$ Clinic and Department of Cardiosurgery

University Clinical Centre Sarajevo

${ }^{3}$ Clinic and Department of Neurosurgery

University Clinical Centre Sarajevo

Corresponding author:

Mirza Halimić

Paediatric clinic

University Clinic Centre Sarajevo

71000 Sarajevo

Bosnia and Herzegovina

halimicm@gmail.com

Tel.: + 38733566450

Fax.: + 38733566525

Received: 1 March 2014

Accepted: 23 September 2014

Copyright (C) 2014 by

Academy of Sciences and Arts

of Bosnia and Herzegovina.

E-mail for permission to publish:

amabih@anubih.ba
Objective. Endocarditis can have profound and devastating neurological consequences, with the vast majority of these complications in patients with left-sided valvular disease. The approach to the acute management of stroke in children with infective endocarditis is limited by the inadequacy of published data on their clinical course and outcome. Case report. This case report presents a 12 year old girl with diagnosed endocarditis, complicated with intracranial hemorrhage, due to the rupture of an aneurysm of the peripheral branch medial cerebral artery and gradient therapeutic approach, with an excellent final result. Conclusion. Congestive heart failure resulting from valvular insufficiency required mitral valve replacement, after cerebral aneurysm clipping.

Key words: Endocarditis, Intracranial hemorrhage, Aneurysm, Treatment.

\section{Introduction}

Approximately $15-40 \%$ of patients with infective endocarditis (IE) eventually require surgery (1). The overall prevalence of hemorrhage in central nervous system involvement of infective endocarditis is 3-7\%. Aneurysms of arteries supplying the brain account for approximately $15 \%$ of the aneurysms occurring in infective endocardi- tis. The term "mycotic aneurysm" describes a mushroom-shaped aneurysm associated with IE (1-3).

Timing of surgery in patients with infective endocarditis and embolic stroke remains controversial, but a report has suggested that surgery can be performed relatively safely within 3 days of the stroke if heart failure is severe; otherwise, a delay of
2-4 weeks is preferable. In patients with associated hemorrhage, a delay of at least 4-6 weeks is preferred (3-5).

The aim of this report is to confirm the validity of a gradient therapeutic approach in a patient with infective endocarditis and intracerebral hematoma, according to the latest European Society of Cardiology (ESC) clinical practice guidelines. 


\section{Case report}

A 12 year old girl with a fever for the two months prior to hospitalization on peroral antibiotic therapy, with prostration, anxiety, screaming, confusion, tachycardia, hallucination and a loud holosystolic murmur on the lower left sternal border, was admitted to the Clinic for Infectious Diseases of the University Clinical Centre Sarajevo, and after initial examination transferred to the Paediatric Clinic. The neurological exam was significant for left leg weakness and diffuse hyper-reflexia of all four extremities.

In the blood culture Streptococcus pneumoniae was detected. Blood studies showed anaemia, a mildly elevated erythrocyte sedimentation rate, as well as $\mathrm{C}$ reactive protein with a normal coagulation panel. Transthoracal ultrasound showed vegetation $(19 \mathrm{x} 11 \mathrm{~mm})$ over the anterior mitral leaflet (Figure 1), with mitral regurgitation, confirmed by CW Doppler. The child was treated with ceftriaxone.

A CT-Scan of the head was interpreted as a left-sided parietal intracerebral hematoma, diameter $30 \mathrm{~mm}$, with compressive oedema on the posterior horn of the lateral ventricle (Figure 2). For technical reasons, digital subtraction angiography (DSA) could not be performed. MRI and MRA brain scan confirmed aneurysm of the peripheral branch of the medial cerebral artery.

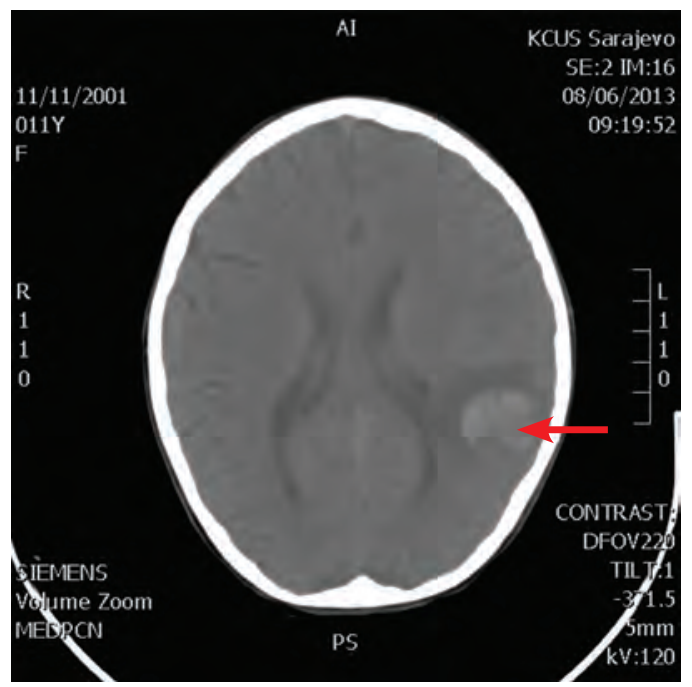

Figure 2 CT scan of the intracranial haemorrhagia (arrow).

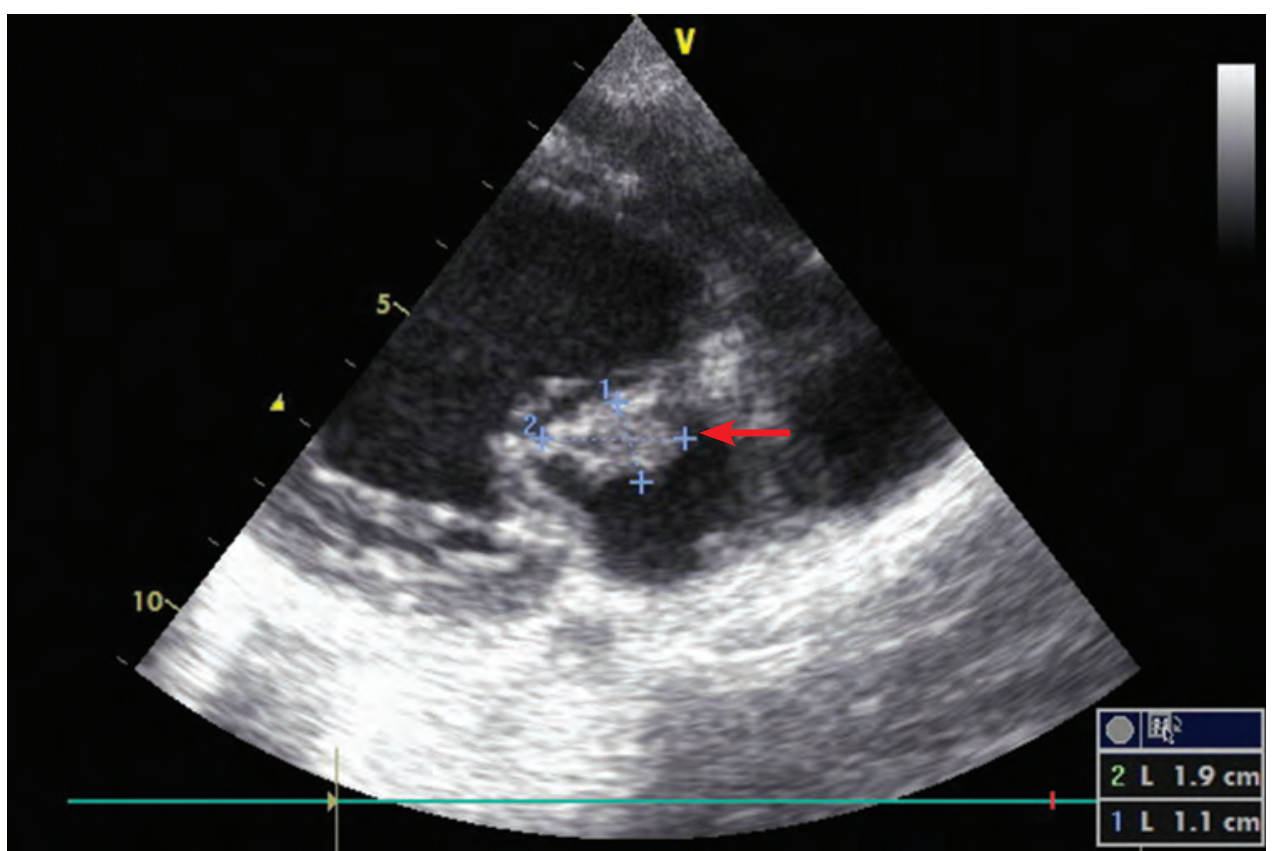

Figure 1 Transthoracic echocardiogram showing vegetation $(19 \times 11 \mathrm{~mm})$ over the anterior leaflet of mitral valve (arrow). 


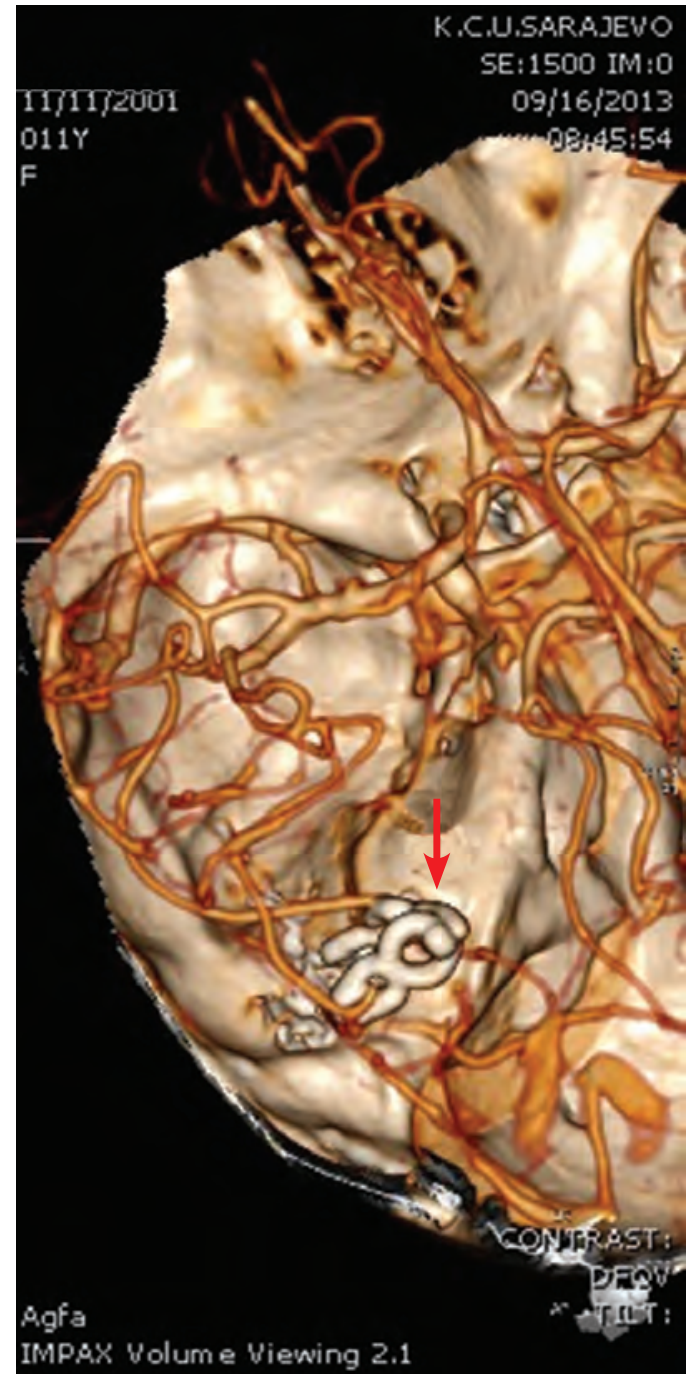

Figure 3 CT angiography scan after cerebral aneurysm clipping (arrow).

Transesophageal echocardiogram (TEE) successfully visualized two mobile vegetations on the atrial side of the mitral valve (anterior leaflet-20x11 $\mathrm{mm}$ and posterior leaflet 18x8mm), with severe mitral and mild tricuspid regurgitation. On control transthoracal and transesophageal echocardiography, after 6 weeks of parenteral antibiotic treatment, significant reduction of the visible vegetation was shown (diameter $6 \times 3 \mathrm{~mm}$ and $4 \times 3 \mathrm{~mm}$ ), with partial prolapse of the A2/P2 segment of the mitral valve, dilatation of the mitral annulus and severe mitral regurgitation (EROA $0.40 \mathrm{~cm}^{2}$, regurgitant volume $62 \mathrm{ml} /$ beat ).

After interdisciplinary paediatric, cardio- and neurosurgical consultation, the decision was made for primary cerebral aneurysm clipping. An interventional radiological approach was not feasible. Surgical treatment was by open craniotomy, multiple clip occlusions and clip reconstruction of the parent vessel, without bypass, as these aneurysms, due to inherent structural characteristics, mainly is not amenable to direct clipping. The aneurysm had poor consistency, was degenerated and had friable walls. Control CT angiography scan showed absence of the aneurysm after clipping (Figure 3). The aneurism had poor consistency, was degenerated and had friable walls. The second postoperative day showed no neurological deficit. Two months after brain surgery the girl was hospitalized again with signs of congestive heart failure, pleural effusion and thoracic drainage. Blood cultures were negative. Transthoracal ultrasound showed a dysmorphic mitral valve, with severe regurgitation, but without proven abscess of the subvalvular apparatus, perforation or chordae rupture. It was not possible to repair the mitral valve and a second relapse required replacement of the mitral valve, with a biological valve prosthesis implantation in the mitral position. Echocardiography showed a small pericardial effusion and reduced cardiac output, FS 16\%, EF 37\%. The child was discharged with anticongestive and anticoagulant therapy, then on further follow up (three months later) she presented with gradually increasing cardiac output, up to the normal range (LVFS 28-32\%, EF 67\%).

\section{Discussion}

Cerebrovascular complications include ischemic or haemorrhagic stroke, transient ischemic attack, silent cerebral embolism, symptomatic or asymptomatic mycotic an- 
eurysm, cerebral abscess, meningitis, toxic encephalopathy and seizure (1-8).

In most cases, the neurologic sequelae are present before the initiation of antimicrobial therapy $(76 \%)$, as in this reported case. One series showed that in patients with infective endocarditis who had CNS involvement, four fifths had ischemic strokes and one fifth had haemorrhages, which is a distribution similar to that of strokes in general.

Aneurysms of arteries supplying the brain account for approximately $15 \%$ of aneurysms occurring in infective endocarditis. Mycotic aneurysms involve the medial cerebral artery territory four times more often than they do the anterior or posterior cerebral arteries. They occur at secondary branches and bifurcations, usually in the lateral fissure near the trifurcation of the medial cerebral artery. When aneurysms are formed, the most likely mechanism is bacterially induced weakening (9).

The onset of valve dysfunction or moderate-to-severe congestive heart failure should lead to an evaluation for immediate valve replacement, which was the case of surgical management in our patient. Cardiac surgery is not contraindicated after an ischemic stroke, a silent cerebral embolism or transient ischemic attack (10).

In patients with neurological complications, the safety of cardiopulmonary bypass has been controversially debated for years. Anticoagulation during cardiac surgery may increase the risk of haemorrhagic transformation of an asymptomatic ischemic stroke. In this case, cardiac surgery should be delayed for 2-3 weeks. If there is a ruptured mycotic aneurysm, it must be resected, clipped or embolised before cardiac surgery (11).

These recommendations for surgery have been made by "The Task Force on the Prevention, Diagnosis, and Treatment of Infective Endocarditis of the European Society of Cardiology" (12). From studies published during the mid-1990s $(4,5,13,14)$, an inter- val of at least 2 weeks between an embolic event and cardiac surgery was recommended. In this reported case, this interval was longer.

Operative mortality is variable but has been reported as $7.6 \%$, with risk factors for death being cardiogenic shock, insidious illness and they increase with age (5). The 9 -year survival rate has been reported to be $71 \%$. Risk factors for death include preoperative neurologic complications $(5,15)$. The risk of neurologic deterioration after valve replacement for infective endocarditis is $20 \%$ in the first 72 hours, $20-50 \%$ 4-14 days postoperatively, less than $10 \%$ beyond 14 days postoperatively, and less than $1 \%$ after 4 weeks (15). In our reported case there was no deterioration during the follow up period after four weeks.

\section{Conclusion}

A multidisciplinary approach in patients with this complex cardio-neurological illness is necessary, concerning the timing of decision making relation to valve surgery for the sake of outcome improvement.

Authors' contributions: Conception and design: $\mathrm{MH}$ and SM; Acquisition, analysis and interpretation of data: $\mathrm{MH}$ and SM; Drafting the article $\mathrm{MH}$ and SM; Revising it critically for important intellectual content: SM, ZB, AK, MK, EO, NH, EB.

Conflict of interest: The authors declare that they have no conflict of interest.

\section{References}

1. Pniitt AA, Rubin RH, Karchmer AW, Duncan GW. Neurologic complications of bacterial endocarditis. Medicine. 1978;57:329-43.

2. Moskowitz MA, Rosenbaum AE, Tyler HR. Angiographically monitored resolution of cerebral mycotic aneurysms. Neurology. 1974;24:1103-8.

3. Habib G, Hoen B, Tornos P, Thuny F, Prendergast B, Vilacosta I, et al. ESC Committee for Practice Guidelines: Guidelines on the prevention, diagnosis, and treatment of infective endocarditis (new 
version 2009): The Task Force on the Prevention, Diagnosis, and Treatment of Infective Endocarditis of the European Society of Cardiology (ESC). Eur Heart J. 2009;19:2369-413.

4. Eishi K, Kawazoe K, Kuriyama Y, Kitoh Y, Kawashima Y, Omae T. Surgical management of infective endocarditis associated with cerebral complications. Multicenter retrospective study in Japan. J Thorac Cardiovasc Surg. 1995;110:174555.

5. Gillinov AM, Shah RV, Curtis WE, Stuart RS, Cameron DE, Baumgartner WA, et al. Valve replacement in patients with endocarditis and acute neurologic deficit. Ann Thorac Surg. 1996;61:1125-9.

6. Morawetz RB, Karp RB. Evolution and resolution of intracranial bacterial (mycotic) aneurysms. Neurosurgery. 1984;15:43-9.

7. Weinstein L. Life-threatening complications of infective endocarditis and their management. Arch Intern Med. 1986;146:953-7.

8. Katz RI, Goldberg HI, Selzer ME. Mycotic aneurysm. Arch Intern Med. 1974;134:939-42.

9. Laguna J, Derby BM, Chase R. Cardiobacterium hominis endocarditis with cerebral mycotic aneurysm. Arch Neurol. 1975;32:638-9.
10. Wilson WR, Geraci JE, Danielson GK, Thompson RL, Spittell JA Jr, Washington JA II, et al. Anticoagulant therapy and central nervous system complications in patients with prosthetic valve endocarditis. Circulation. 1978;57:1004-7.

11. Nakahara I, Taha M, Higashi T. Different modalities of treatment of intracranial mycotic aneurysms: report of 4 cases. Surg Neurol. 2006;66:405-9.

12. Peters PJ, Harrison T, Lennox JL. A dangerous dilemma: management of infectious intracranial aneurysms complicating endocarditis. Lancet Infect Dis. 2006;6:742-8.

13. Chapot R, Houdart E, Saint-Maurice JP, Aymard A, Mounayer C, Lot G, et al. Endovascular treatment of cerebral mycotic aneurysms. Radiology. 2002;222:389-96.

14. Gillinov AM, Shah RV, Curtis WE, Stuart S, Cameron DE, Baumgartner WA, et al. Valve replacement in patients with endocarditis and acute neurologic deficit. Ann Thorac Surg. 1996;61:1125-30.

15. Angstwurm K, Borges AC, Halle E, Schielke E, Einhäupl KM, Weber JR. Timing the valve replacement in infective endocarditis involving the brain. J Neurol. 2004;251:1220-6. 